\title{
Superconducting phonon spectroscopy using a low-temperature scanning tunneling microscope
}

\author{
H. G. LeDuc, W. J. Kaiser, B. D. Hunt, and L. D. Bell \\ Jet Propulsion Laboratory, California Institute of Technology, Pasadena, California 91109 \\ R. C. Jaklevic \\ Ford Motor Company, Dearborn, Michigan 48121-2053 \\ M. G. Youngquist \\ California Institute of Technology, Pasadena, Calfornia 91125
}

(Received 29 September 1988; accepted for publication 15 Decenber 1988)

\begin{abstract}
We report the first observation of phonon density of states effects in a superconductor using a low-temperature scanning tunneling microscope (STM). The phonon effects were observed using a $S T M$ spectroscopy method to measure $d l_{\text {tanneling }} / d V$ vs $V$ for the tunnel junction formed by the Au STM probe and a superconducting $\$ \mathrm{~b}$ sample.
\end{abstract}

The scanning tunneling microscope (STM), since its invention, ${ }^{1}$ has evolved into a sophisticated tool for direct imaging of many surfaces with atomic resolution. More recently, the STM has emerged as a powerful spectroscopic tool with the potential for observation of surface and subsurface electronic properties also with very high spatial resolution. The large field of conventional tunneling spectroscopy on macroscopic tunnel junctions is credited with many fundamental observations. Measurements involving macroscopic tunnei junctions, however, are limited by the spatial averaging over the junction area and potential insulator barrier induced alteration of the system under study. Due to the local nature of the tunnel current in a STM experiment, one can hope to study macroscopically nonideal samples such as polycrystalline thin films and measure properties of fundamental as well as technological importance.

Historically, tunneling spectroscopy has been the most sensitive probe of the superconducting state. Observation of the superconductor energy gap by current-voltage (I-D) spectroscopy using the STM has been reported. ${ }^{2}{ }^{4}$ In this letter we report, for the first time, the application of the STM to the observation of superconductor phonon density of states effects in conductance-voltage $(d I / d V-V)$ spectroscopy. Conductance spectroscopy has been important in the study of superconductors. In the case of a normal metalinsulator-superconductor (NIS) tunnel junction, the normalized conductance as a function of bias voltage, $\sigma(\mathrm{eV})=G_{n s}(\mathrm{eV}) / G_{n n}(\mathrm{eV})$ where $G_{n s}$ and $G_{n n}$ are the tunneling conductance with the $S$ eiectrode in the superconducting and normal states respectively, is a neariy exact rep. resentation of the superconductor excitation density of states. Small structures in the excitation density of states deviating from the predictions of the Bardeen-CooperSchrieffer (BCS) theory ${ }^{5}$ were first observed by Giaever ${ }^{6}$ using conductance-voltage spectroscopy with macroscopic area tunnel junctions. The deviations are strongest in superconductors such as $\mathrm{Pb}$, and were used to establish the validity of the strong coupling modifications of the theory of superconductivity culminating in the theory of Eliashberg. ${ }^{7}$ This structure has been showr to arise from the energy dependence of the phonon mediated electron-electron coupling responsible for the superconducting state. The devia- tions from $\mathrm{BCS}$ behavior in $\mathrm{Pb}$ observed in conductance-voitage spectroscopy are weak; the phonon structure is resolved as a change in conductance of oniy a few percent of the total conductance. Therefore, the observation of phonons represents a difficult measurement for low-temperature STM where the tunnel current and conductance signals are reduced by a factor of greater than $10^{6}$ compared to conventional macroscopic area tunnel junctions.

Our STM system has been described previously. ${ }^{4}$ The basic design is similar to one used for room-temperature STM studies ${ }^{8}$ with modification for use in low-temperature STM of superconductors. For phonon spectroscopy the tunnel voltage range of interest is $0.0-20.0 \mathrm{mV}$. The need to maintain large tunnel resistances in STM spectroscopy has been discussed previously. Tunneling resistance for these measurements was maintained in the $1 \times 10^{7}-1 \times 10^{9} \Omega$ range. Under these conditions the tunneling currents are typically in the range of $20-2000 \mathrm{pA}$. Two basic requirements for the feedback and spectroscopy method are (1) small voltage control and (2) direct $d I / d V$ measurement. To meet these requirements techniques used in macroscopic tunneling spectroscopy were employed, two tunnel voltage modulation signals at separate frequencies $\left(f_{0}\right.$ and $\left.f_{\mathrm{i}}\right)$ are appiled simuitaneously to the STM tunnel junction. The tipsample separation control is achieved, using techniques similar to those employed in Ref. 2, by measuring the amplitude of the current signal at the lower frequency $f_{0}$ with a lock-in ampiffier and maintaining this amplitude at a constant value using a feedback circuit. Using this method, the $I-V$ spectra can be measured by monitoring the tunnel voltage and current without interrupting feedback control. The $d I / d V-V$ spectra were measured using standard analog derivative techniques by phase sensitively detecting the modulated current at the higher modulation frequency $f_{1}$ using a second lock-in amplifier. To avoid distortions due to slew rate limitations of the second lock-in, it is important to keep $f_{0}$ as low as possible while maintaining stable tunneling. In the experiments reported here $f_{0}=10-20 \mathrm{~Hz} .1 . V$ spectra were measured as a function of tunneling resistance and have been shown to be independent of tunneling resistance for resistances of $10^{7} \Omega$ or greater. The sweep frequency $f_{0}$ was also varied to check for distortions due to insufficient electronic 
bandwidth. In this regard, the preamplifier incorporated into our STM design is critical. The spectra were acquired by a signal averager to improve the signal to noise ratio.

The superconducting materials were thin films deposited on silicon. The pb samples were thermally evaporated from $99.999 \%$ pure metal in a iquid-nitrogen trapped diffusior-pumped vacuum chamber. The NbN was deposited by reactive de magnetron sputtering from a high-purity $\mathrm{Nb}$ karget in an argon-nitrogen atmosphere in a high vacuum chamber under conditions used for making NbN-based tunnel junctions. ${ }^{9}$

A topogram of $\mathrm{NbN}$ taken at $4.2 \mathrm{~K}$ using the low-temperature STM is shown in Fig. 1. The surface reveals a single-crystal grain with a sequence of two atom layer high steps. Topograms such as this can be rescanned over periods of many hours with minimal drift in the scan window. This stability is required in the derivative spectroscopy experiments where extensive signal averaging is need to enhance the signal-to-noise ratio.

Shown in Fig. 2 is an electron tunneling $1-V$ spectrum for $\mathrm{NbN}$ taken with a STM at $4.2 \mathrm{~K}$. The data exhibit NIS character with the characteristic superconductor energy gap clearly defined. The small conductance region followed by a sharp rise in the current at one half the gap voltage and an asymptotic approach to the normal-state tunneling characteristic clearly distinguishes NIS turneling from other tunneling $I-V$ characteristics. The theoretical $I-V$ data can be numerically calculated using an elementary tunneling formalism and BCS density of states. ${ }^{10}$ Using a single parameter $\Delta$ and approximating the normal conductance from the data gives $\Delta=2.58 \mathrm{meV}$, which is in the range expected for $\mathrm{NbN} .11,12$

An $F-I$ spectra obtained by electron tunneling into $\mathrm{Pb}$ at $4.2 \mathrm{~K}$ using the low-temperature STM is shown in Fig. 3. The siight hysteresis in the STM spectrum is a result of the bidirectional voltage sweep and the resulting tip-sample displacement current. A fit yields $\Delta=1.28 \mathrm{meV}$ for this data. Using the normalized temperature dependence of the energy gap measured by $\mathrm{Adler}{ }^{13}$ and our fit data at $4.2 \mathrm{~K}$ we have

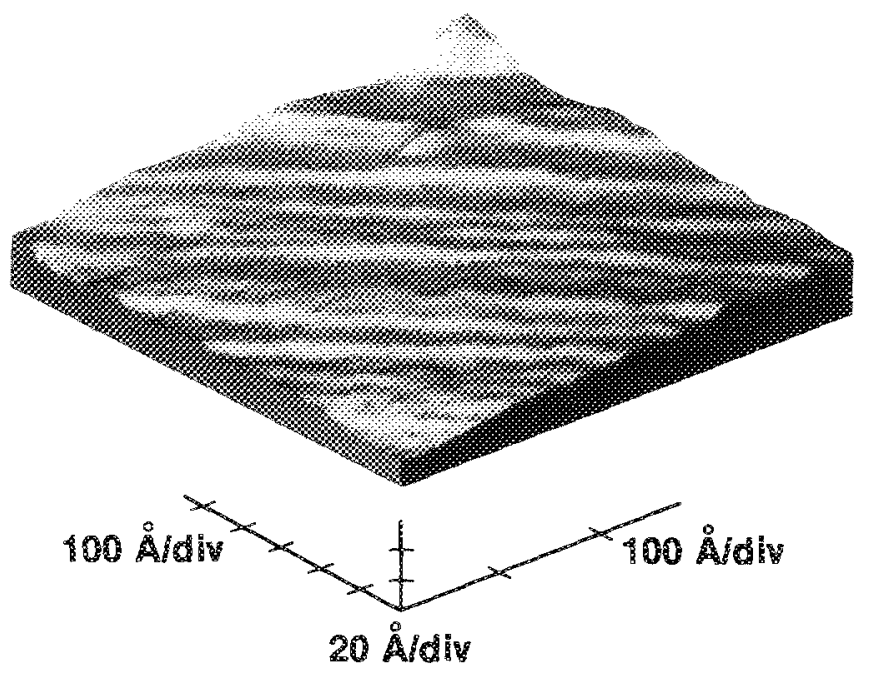

FhG. 1. STM topogram of a sputter-deposited NbN thin alm obtained at $4.2 \mathrm{~K}$.

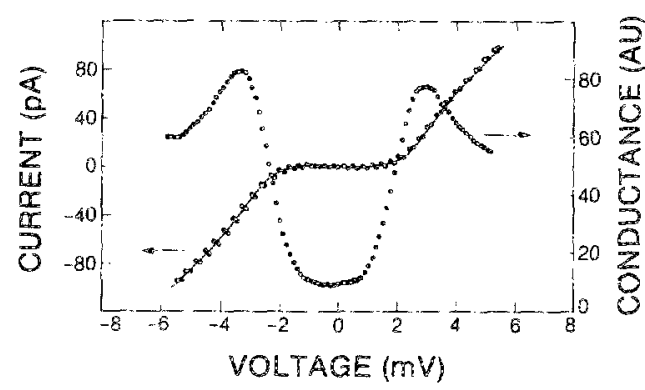

FIG. 2. Electron tunneling current-voltage and conductance-voltage spectra of a $\mathrm{NBN}$ thin film measured by STM a $4.2 \mathrm{~K}$. The points are STM data while the line represents a theoretical fit.

calculated a zero termperature gap parameter $\Delta_{0}=1.36 \mathrm{meV}$ which is within the range observed for $\mathrm{Pb}{ }^{14}$ The smaller energy gap of $\mathrm{Pb}$ relative to $\mathrm{NbN}$ leads to clearly observable changes in the character of this spectrum from that of $\mathrm{NbN}$ shown in Fig. 2. The energy gap difference is refiected in the smailer extent of the lower conductance region. In addition, athough the subgap current is dominated by the thermal broadening in the normal metal (the energy gap of $\mathrm{Pb}$ at 4.2 $\mathrm{K}$ is large compared to $k T$, the extent of the smearing as a fraction of the energy gap leads to a less dramatic I- $V$ nonlinearity.

For NES tunneling in macroscopic turnel junctions, the phonon effects occur for voltage bias above one half of the superconductor energy gap and the major siructure in the conductance is observed below $13 \mathrm{meV}$ in $\mathrm{Pb}$. It can be seen from the $\mathrm{Pb} \mathrm{I}-\mathrm{V}$ curve in Fig. 3 that the deviations from linearity above the gap are small and derivative spectroscopy is, therefore, required to resolve them. Conductance measurements over the voltage range of interest are shown in Fig. 4 along with that measured using a macroscopic $\mathrm{Pb} / \mathrm{AlO}_{x} / \mathrm{Al}$ tunnel junction ${ }^{15}$ for comparison. ${ }^{16}$ There is good qualitative agreement between the experimental STM and macroscopic tumnel junction curves. The conductancevoltage spectra measured by STM covering the superconductor gap voltage range for a $\mathrm{NbN}$ thin film are shown in Fig. 2. Phonon density of states effects in $\mathrm{NbN}$ are weaker and broader and they were not observed with our STM.

Recently it has been suggested that multiparticle tunneling ${ }^{17}$ which is predicted to give rise to excess subgap tunneling current might be operative in STM experiments per-

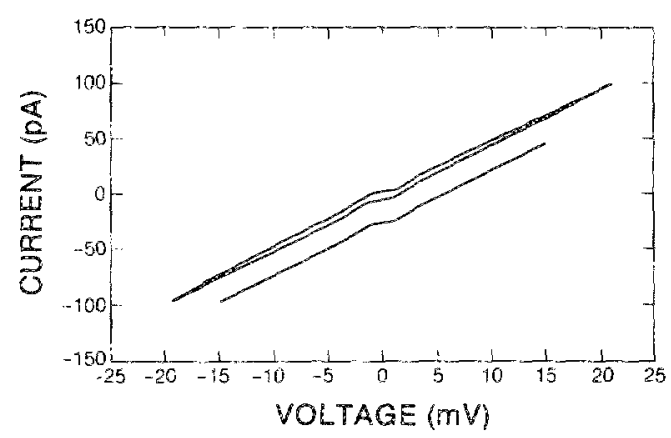

FIG. 3. Electron vunneling $F-V$ spectra of a $\mathrm{Pb}$ thin film measuredby $\mathrm{STM}$ at $4.2 \mathrm{~K}$ (upper curve) and a theoretical fit displaced for clarity (lower curve). 


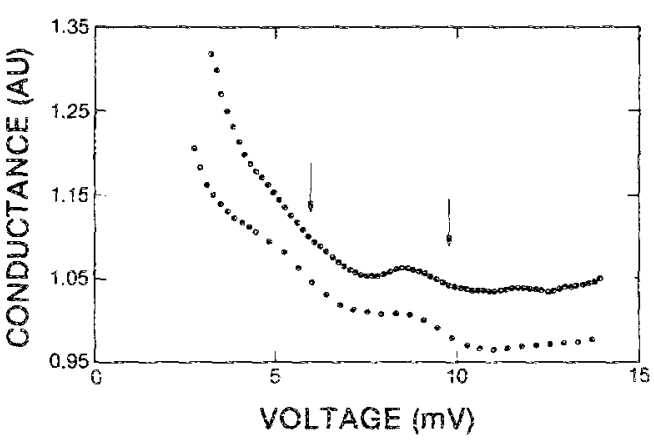

FIG. 4. Tunneling conductance-voltage spectra obtained at $4.2 \mathrm{~K}$ for a $\mathrm{Pb}$ thin film. The upper curve is measured by STM electron tunneling and the lower measured on a macroscopic $\mathrm{Pb} / \mathrm{AlO} \mathrm{O}_{x} / \mathrm{Al}$ turnel jusetion. The arrows indicate features associated with the transverse (left arrow) and longitudinal (right arrow) peaks in the phonon density of states.

formed at low tunneling resistance, ${ }^{18}$ Careful observation of the $I-V$ characteristics in the gap region of $\mathrm{NbN}$ did not reveal any excess tunneling current. The BCS-based fit ${ }^{10}$ to the $\mathrm{NbN} I-V$ spectrum in Fig. 2 coes not inciude multiparticle tumneling, yet accounts for the measured subgap currents within experimental error.

In the course of our experiments, we have observed variations in the superconductor energy gap in $I-V$ measurements of $\mathrm{Pb}$ thin films from region to region on the same sample and from sample to sample. One possible explanation for this observation is the known gap anisotropy of $\mathrm{Pb}^{19}$ combined with the polycrystalline nature of the deposited films and the local nature of the STM tunnel probe. Exper:mental zero temperature gap parameter values $\left(\Delta_{0}\right)$ reported in the literature for single-crystal samples vary from 1.18 to $1.40 \mathrm{meV} .{ }^{19}$ This range includes variations due to direction-dependent gap anisotropy and variations due to mult:ple energy gap superconductivity arising from different sheets of the Fermi surface. These gap variations are masked in macroscopic tunnel junctions fabricated with polycrysta!line films due to spatial averaging, which again highlights the potential of STM to measure fundamental properties on samples which are macroscopically nonideal, such as polycrystaline thin-film samples.

The observation of phonon effects in superconductors represents a measurement of conductance to a minimum of one part in one hundred. This measurement demonstrates that, in principle, variations in phonon effects could be spatially imaged with a STM. However, measurement of low noise spectra with small currents requires extensive signal averaging and limits the spatial resolution with which these variations could be observed in an experiment of :easonable duration. The advantages of STM for conductance spectroscopy may lie in the formation of a microscopic, nearly ideal tunnel junction on samples which are macroscopically noni- deal such as polycrystalline thin films. Thus local properties of samples can be measured at selected points of the sample surface.

In summary, we have developed techniques for simultaneous tip-sample separation control and $I-V$ and $d I / d V-V$ measurement. Using these techniques we have made the first observation of phonon density of states spectrum in a superconductor using a STM. We have measured electron tunneling $I-V$ characteristics for $\mathrm{NbN}$ and $\mathrm{Pb}$. We have observed variations in the superconductor energy gap in $I-V$ measurements of $\mathrm{Pb}$ thin films. One possibie explanation for this observation is gap anisotropy observed for $\mathrm{Pb}$ combined with the polycrystalline nature of the deposited films and the local nature of the STM tunnel probe. In addition, our $I-V$ measurements on $\mathrm{NbN}$ and $\mathrm{Pb}$ films under typical STM conditions showed no evidence for multiparticie tunneling effects.

This work was performed at the Jet Propulsion Laboratory, California Institute of Technology, as part of the Center for Space Microelectronics Technology and was sponsored by the Strategic Defence Initiative Organization/Innovative Science and Technology through an agreement with the National Aeronautics and Space Administration (NASA).

G. Binnig and H. Rohrer, IBM Res. Devclop. 30, 355 (1985)

${ }^{2}$ A.L. de Lozanne, S. A. Elrod, and C. F. Quate, Phys. Rev. I.tt. 54, 2433 (1985).

${ }^{3}$ J. R. Kirtley, S. I. Raider, R. M. Feenstra, and A. F. Fein, Appl. Phys. Lett. 50, 1607 (1987).

${ }^{4}$ II. G. LeDuc, W. J. Kaiser, and J. A. Stern, Appl. Phys. Lett. 50, 1921 (1987).

5J. Bardeen, L. N. Cooper, and J. R. Schrieffer, Phys. Rev. 99, 1140 (1955)

'I. Giaever, IH. R. Hart, and K. Mergle, Phys. Rev. 126, 941 (1962).

${ }^{7}$ G. M. Eliashberg, Sov. Phys. JETP 11, 696 (1970).

${ }^{8}$ W. J. Kaiser and R. C. Jaklevic, Rev. Sci. Instrum. 59, 537 (1988).

${ }^{9}$ H. G. LeDuc, I. A. Stern, S. Thakoor, and S. K. Khanna, IEEE Trans. Magn. MAG-23,863(1986).

S. Shapiro, P. H. Smith, J. Nicol, J. L. Miles, and P. F. Strong, IBM J. Res. Dev. 6, 34 (1962)

"M. R. Beasley and C. J. Kircher, in Superconducting Materials Science, edited by S. Foner and 13. B. Schwartz (Plenum, New York, 1981), p. 661.

${ }^{12}$ M. Gurvitch, J. P. Remeika, J. M. Rowell, J. Geerk, and W. P. Lowe, IEEE Trans. Magn. MAG-17, 509 (1985).

${ }^{13}$ J. G. Adler and T. T. Chen, Solid State Commun. 9, 1961 (1971).

${ }^{34}$ W. L. McMillan and J. M. Rowelt, Phys. Rey. Lett. 14, 108 (1965).

${ }^{15}$ R. C. Jaklevic (unpublished).

"To make qualitative comparisons, the STM data were translated without dilation along the voltage axis to remove experimental voltage offsets.

17. R. Schrieffer and J. W. Wilkins, Phys. Rev. Lett. 10, 17 (1963).

${ }^{18}$ N. Garcia, F. Flores, and F. Guinea, 3. Vac. Sci. Technol. A 6, 323 (1988).

${ }^{9}$ For a discussion of gap anisotropy in superconductors, see E. L. Wolf, Principles of Electron Tunneling Spectroscopy (Oxford University, New York, 1985). 